\title{
Editorial
}

\section{Monseñor Piñera, Carl Wiggers y Claude Beck, y la Fibrilación y Desfibrilación Ventricular Un homenaje al último Padre Conciliar y Primer Fisiólogo Cardiovascular de Chile.}

\author{
Ricardo Zalaquett S. \\ Cirujano Cardiovascular \\ Profesor Titular \\ Pontificia Universidad de Chile \\ Editor Revista Chilena de Cardiología
}

Monseñor Bernardino Piñera Carvallo (Figura 1), último sobreviviente del Concilio Vaticano Segundo, falleció meses antes de cumplir 105 años, el domingo 21 de junio de 2020. Sabido es que Monseñor Piñera fue médico de profesión, que ingresó a estudiar medicina a la Universidad Católica de Chile y que, como era en ese entonces, se recibió de médico en la Universidad de Chile, en 1940. También es sabido que terminando su carrera hizo una estadía en Estados Unidos. Pero, lo que no es bien sabido, es que esta estadía correspondió a una beca de la Oficina Sanitaria Panamericana "a quien debo la oportunidad de haber ido a los Estados Unidos, y que me dieron las facilidades necesarias para poder dedicarme al trabajo experimental durante mi estadía allá", como lo dejó establecido en su "Tesis de prueba para optar al título de médico-cirujano de la Universidad de Chile" (Figura 2). Pero, lo que es menos sabido aún es que esta estadía la realizó en el Departamento de Fisiología de la Escuela de Medicina de la Western Reserve University, en Cleveland, Ohio y que dirigía Carl J. Wiggers.

Carl J. Wiggers (Figura 3) es uno de los fisiólogos cardiovasculares de mayor prestigio de todos los tiempos.

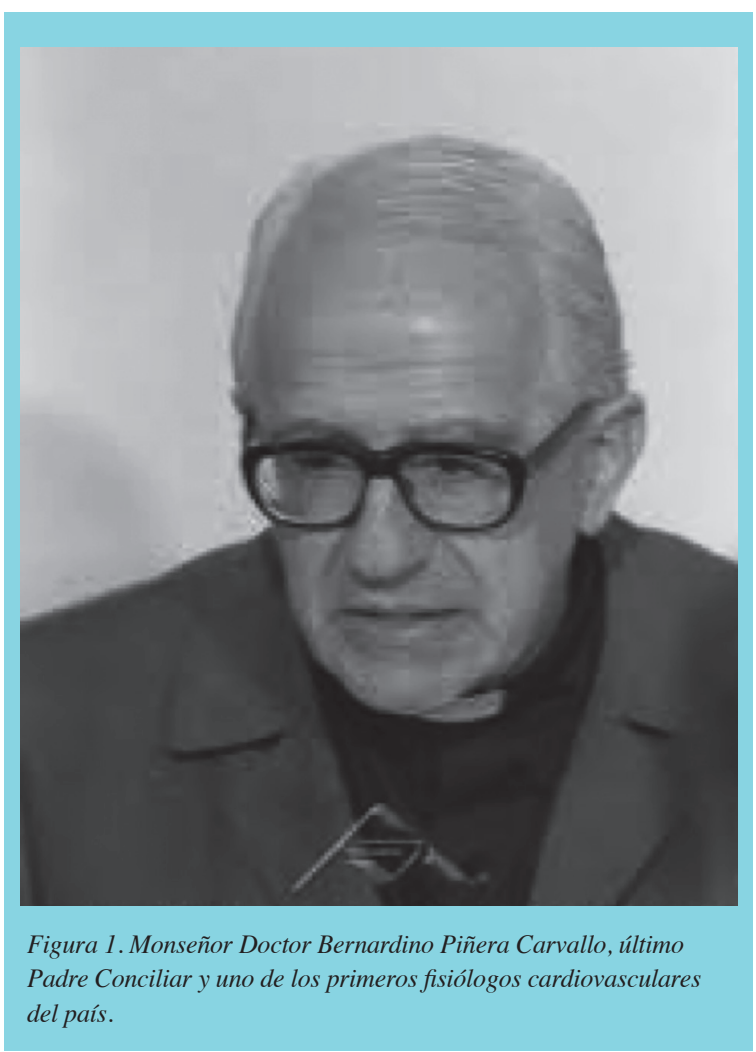

Correspondencia:

Dr. Ricardo Zalaquett S.

rzalaque@med.puc.cl 


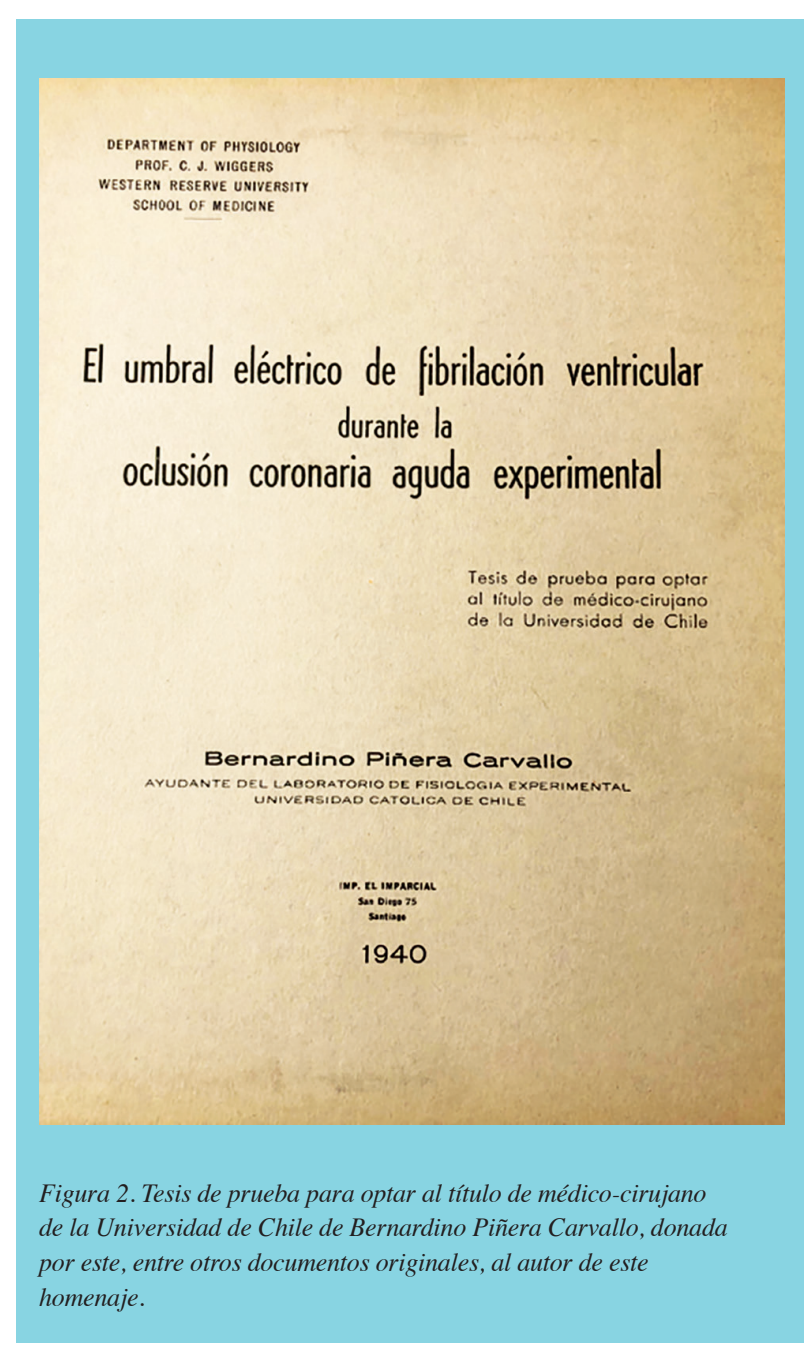

Fue Jefe del Departamento de Fisiología de la Escuela de Medicina de la Western Reserve University, actualmente, Case Western Reserve University, en Cleveland, Ohio, entre 1918 y 1953. Entre sus contribuciones más importantes se cuentan el desarrollo de técnicas para registrar la presión en el ventrículo izquierdo y en la sangre, entender las consecuencias hemodinámicas de las valvulopatías y del shock, entre otras; y alcanzó la fama al desarrollar el "Diagrama de Wiggers", el que permite comprender todo el Ciclo Cardíaco, integrando en éste la presión aórtica, ventricular y auricular, el volumen ventricular y el electrocardiograma. Pero, sin duda, su contribución clínica más importante fue el desarrollo de técnicas de resucitación que se pudiesen aplicar en casos de muerte en el pabellón de operaciones, lo que no era infrecuente en los tiempos del cloroformo, junto al cirujano cardiovascular, Claude Beck, de la misma institución ${ }^{1}$. Entre muchos logros

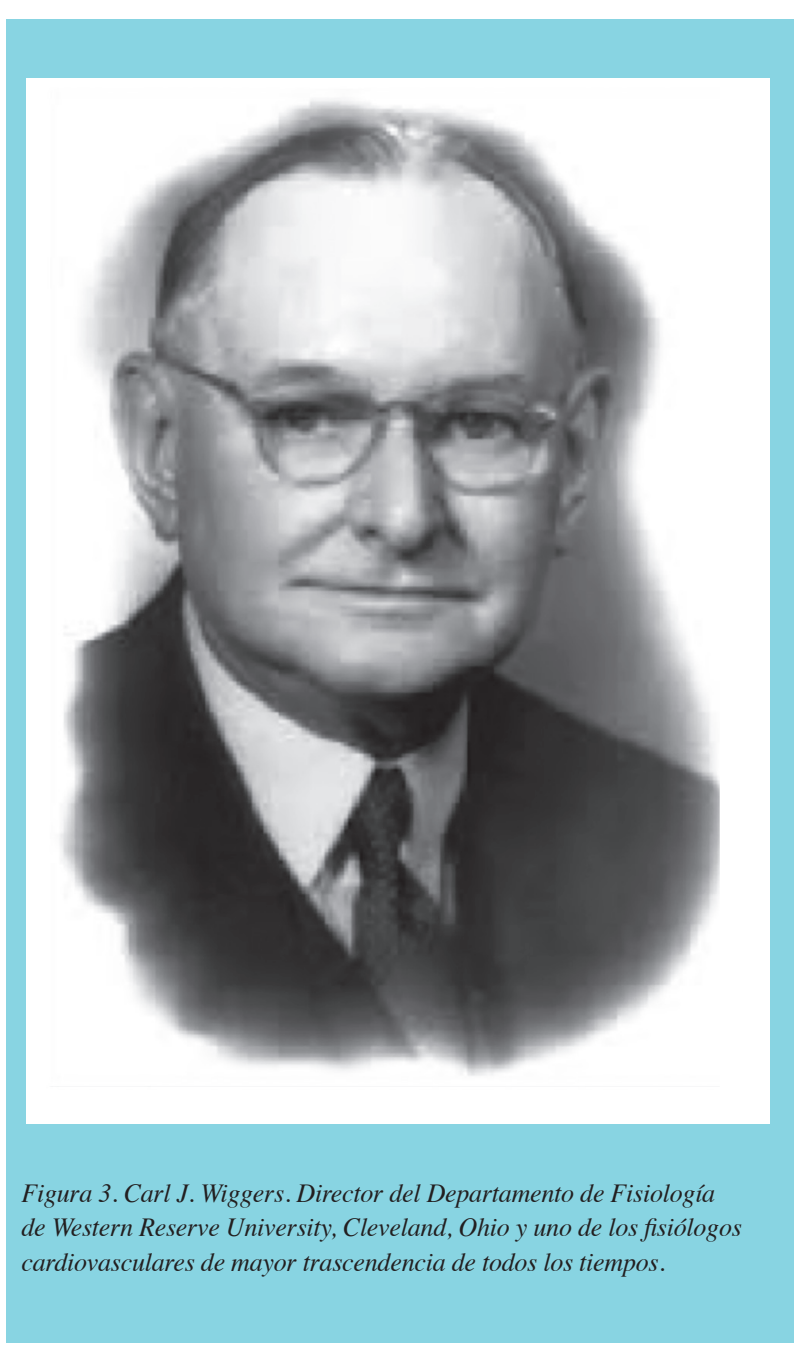

y reconocimientos, Wiggers fue el fundador y primer editor de Circulation Research, en 1953.

La fibrilación y desfibrilación ventricular. En 1850, Carl Ludwig, en Leipzig, demostró que era posible inducir, en el animal de experimentación, fibrilación ventricular aplicando corriente eléctrica al corazón y en 1879, otro alemán, Hugo von Ziemssen, demostró en un paciente sometido a una resección de un cáncer de la pared torácica, cuyo corazón quedó expuesto, que era posible alterar la frecuencia y el ritmo cardíaco aplicando corriente eléctrica, tanto directamente al corazón como a través de la pared torácica. En 1887 , John MacWilliam, en la Universidad de Aberdeen, Escocia, efectuó una serie de experimentos en fibrilación ventricular, los que le permitieron caracterizar a esta "contracción fibrilar" como una condición en la que "el músculo ventricular cae en un estado de con- 
tracción arrítmica irregular mientras hay una gran caída de la presión arterial. Los ventrículos se dilatan con sangre puesto que el rápido temblor de sus paredes es insuficiente para expulsar su contenido; la acción muscular es el resultado de una contracción rápida descoordinada del tejido muscular"1.

Si bien el interés principal de MacWilliam era dilucidar los mecanismos básicos de la electrofisiología cardíaca, tenía clara conciencia de la potencial aplicación práctica de sus observaciones. En 1887 comunicó los resultados de sus experimentos, los que demostraban que la contractilidad cardíaca podía ser restituida por medio de descargas eléctricas de moderada intensidad. Pocos años más tarde, MacWilliam estableció la importancia de la fibrilación ventricular como causa de muerte súbita y su relación con la isquemia miocárdica. También estableció, muy claramente, las diferencias entre el paro cardíaco por asistolia del producido por fibrilación ventricular. Sin embargo, estas observaciones experimentales de MacWilliam en su tiempo no se extendieron a la práctica clínica por que no existían los métodos que permitieran documentar el mecanismo del paro cardíaco en los seres humanos. Fue necesario esperar hasta fines del siglo XIX para que Willem Einthoven inventara el electrocardiógrafo y hasta comienzos del siglo XX para que Augustus Hoffman registrara electrocardiográficamente la fibrilación ventricular. Sin embargo, el registro clínico de la fibrilación ventricular tuvo que esperar al pionero de la electrocardiografía clínica, Thomas Lewis, si bien esto continuo siendo inusual, dadas las características de la fibrilación ventricular, que lleva a la muerte súbita e inesperada ${ }^{1}$.

En 1899, Prevost y Battelli, en Ginebra, observaron, experimentalmente, que era posible convertir la fibrilación ventricular a ritmo regular con una corriente eléctrica. Sin embargo, tomo mucho tiempo para que se llegara a la desfibrilación de un corazón humano, puesto que el interés al respecto, y a pesar de comunicaciones de distintos grupos, fue paulatinamente decayendo ${ }^{1}$.

De este modo, en los años 30 y 40 del siglo pasado, la única institución en Estados Unidos dedicada seriamente a la resucitación cardiaca era Western Reserve University, en Cleveland, Ohio, en la que ya existía un interés al respecto, desde los primeros años del siglo XX. En 1918 Carl J. Wiggers asumió la dirección del Departamento de Fisiología de la Escuela de Medicina de Western Reserve University, reemplazando a un antiguo discípulo de MacWilliam, J.J.R. Macleod, quien

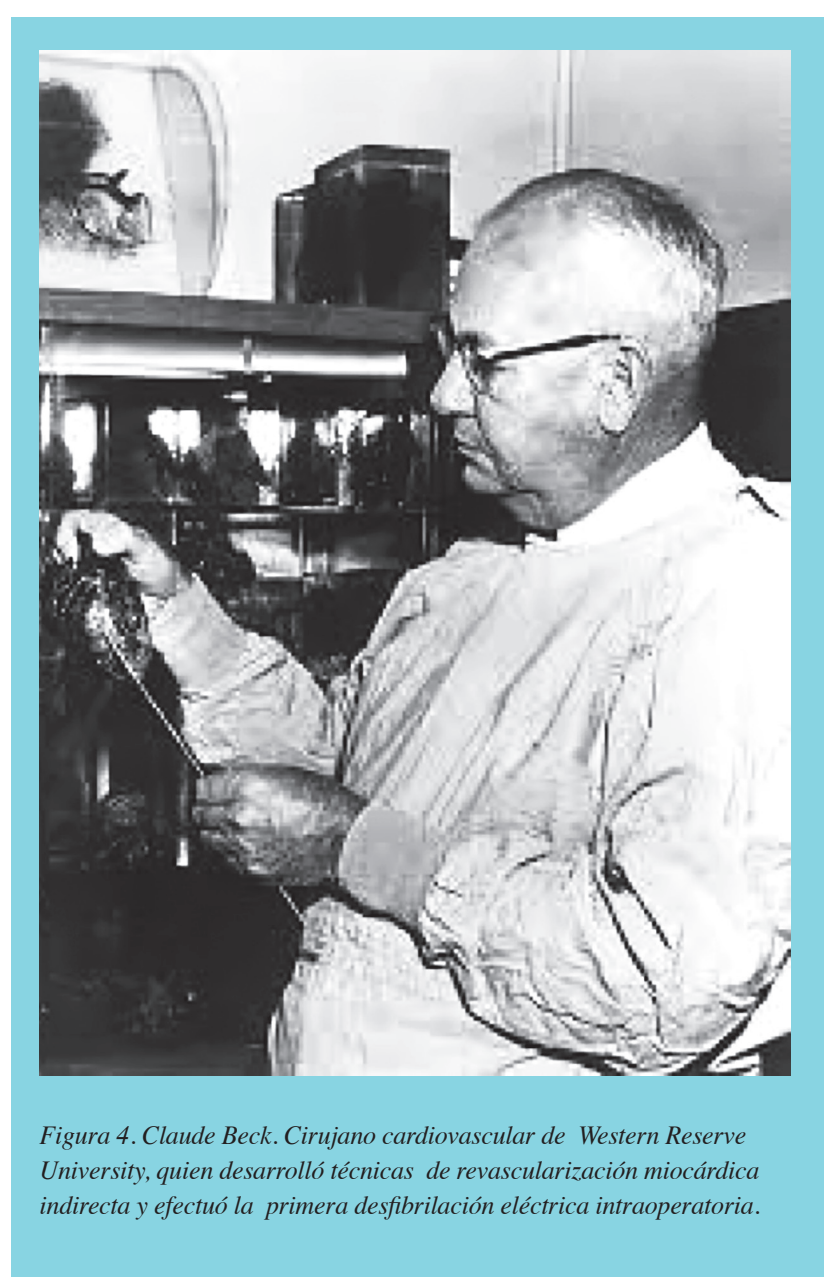

en 1923 ganaría el Premio Nobel como co-descubridor de la insulina. Ya en los años 30 Wiggers era considerado el fisiólogo cardiovascular líder de Estados Unidos, habiendo publicado numerosos artículos y dos importantes monografías sobre fisiología del sistema cardiovascular. Entre estos, Wiggers publicó una serie de trabajos sobre fibrilación ventricular en perros, en los que trataba de convertirlos a ritmo sinusal con inyecciones de cloruro de potasio y cloruro de calcio, junto con masaje cardiaco directo. Wiggers había decidido inicialmente no persistir en la línea de Prevost y Battelli en cuanto a la cardioversión eléctrica puesto que la idea le parecía "fantasiosa". Sin embargo, posteriormente se interesó en esta, combinándola con la compresión cardiaca manual, sosteniendo que el "método debiera servir en la resucitación del corazón humano expuesto durante una cirugía cardiaca en la que se produjera una fibrilación ventricular accidental". El riesgo de muerte súbita durante la anestesia con cloroformo era conoci- 
do desde la introducción de este anestésico en 1847, pero el mecanismo de esta era motivo de controversia, lo que se mantuvo hasta 1911 en que A.G. Levy, anestesista del Hospital Guy de Londres, estableció, junto a Thomas Lewis, usando la electrocardiografía, que la causa era la fibrilación ventricular. ${ }^{1,2}$.

Claude Beck (Figura 3), originalmente un neurocirujano, en Western Reserve University se interesó posteriormente en el tratamiento del Ángor Pectoris, desarrollando distintos procedimientos quirúrgicos indirectos para aumentar la irrigación del corazón, basados en producir una pericarditis estéril por abrasión mecánica o química de ambas hojas pericárdicas, con el objeto de producir, a su vez, adherencias vascularizadas que se comunicaran con los vasos miocárdicos, en los años treinta ${ }^{3}$. En 1937 Beck manifestó su interés en los estudios recientes de su colega en Western Reserve University, Carl Wiggers, en relación a la fibrilación y desfibrilación ventricular, y, meticulosamente comenzó a definir los pasos que seguiría para tratar la fibrilación ventricular intraoperatoria con medicamentos y descarga eléctrica, publicando en 1941 dos casos no exitosos de intento de resucitación. En 1947 Beck comunicó el primer caso exitoso de un paciente sobreviviente a una fibrilación ventricular tratada con desfibrilación eléctrica. Esto despertó un inusitado interés práctico en las técnicas de resucitación de Wiggers y Beck, los que organizaron una serie de cursos al respecto, los que llevaron a Cleveland a más de 3000 médicos y cirujanos! Claude Beck sostuvo y promovió que en todo pabellón de operaciones debería haber un aparato de desfibrilación cardiaca eléctrico. En los años 50, Paul Zoll, en Boston, desarrolló, finalmente, la cardioversión eléctrica de la fibrilación ventricular con el tórax cerrado ${ }^{1-3}$. A mediados de 1939, Bernardino Piñera Carvallo, llegó a Cleveland para incorporarse como fellow al "Department of Physiology, Western Reserve University, School of Medicine", que dirigía Carl J. Wiggers. En 1940 Piñera publicó, junto a Wiggers, en el American Journal of Physiology, el artículo titulado "The effects of myocardial ischemia on the fibrillation threshold" - The mechanism of spontaneus ventricular fibrillation following coronary occlusion, resultado de su trabajo experimental durante su beca en dicho departamento ${ }^{4}$.

Estos descubrimientos también fueron la base para su "Tesis de prueba para optar al título de médico-cirujano de la Universidad de Chile" (Figura 2), que rindió el 7 de octubre de 1940, y que aprobó con 20 puntos, de un máximo de 21 (mínimo12). La Comisión que prestó su aprobación a la Memoria del señor Bernardino Piñera Carvallo estuvo compuesta por los siguientes Profesores:

Dr. Eduardo Cruz Coke, Profesor Titular de Química Biológica.

Dr. Francisco Hoffman, Profesor Titular de Fisiología. Dr. Jorge Mardones R., Profesor Extraordinario de Farmacología.

(Eduardo Cruz Coke y Jorge Mardones fueron ministros de "Salud Pública y Previsión Social"; el primero fue, además, candidato a presidente de la República y el segundo, "Premio Nacional de Ciencias". Francisco Hoffman fue el fundador del Instituto de Fisiología de la Universidad de Chile).

\section{Las conclusiones a las que llegó el tesista Bernardi- no Piñera fueron las siguientes:}

1. La oclusión aguda experimental del "ramus descendens anterior" de la arteria coronaria izquierda, determina en muchos casos la aparición de estímulos ectópicos que perturban el ritmo cardíaco hasta producir en ciertos casos la fibrilación espontánea de los ventrículos. Este hecho es sobradamente conocido de fisiólogos y de clínicos, y se nota tan solo por cuanto representó un obstáculo en muchos de los experimentos.

2. En ciertos casos, estos estímulos ectópicos tardan en aparecer o son escasos. Tales casos se presentan para poner en evidencia un descenso franco del umbral eléctrico de fibrilación ventricular, que dura y probablemente se acentúa mientras se mantiene la oclusión. Cuando se restablece la circulación, el umbral vuelve a su valor primitivo.

3. Llama la atención el hecho de que ciertos perros reaccionaban ante la oclusión con estímulos ectópicos y fibrilación espontánea, mientras otros no presentaban dichos estímulos y fibrilaban solo bajo la acción de los eléctricos; y los unos y los otros mantenían el mismo tipo de respuesta en las oclusiones sucesivas. Esto puede atribuirse, se cree, a factores propios de cada experimento: el estado previo del miocardio del perro y el grado de eficacia de la oclusión.

4. Se recalca el hecho que, si bien en ciertos experimentos la aparición espontánea de la fibrilación ventricular no permitió determinar el umbral de fibrilación 
durante la oclusión coronaria, esto no significa que el umbral no hubiera descendido; antes bien es lógico suponer que lo hizo y que la fibrilación espontánea observada en estos casos fue favorecida por tal descenso. Al año siguiente, Bernardino Piñera Carvallo ingresó al Seminario Mayor de Santiago, para ordenarse como sacerdote en 1948. Como seminarista, continuó partici- pando en el Laboratorio de Fisiología Experimental de la Universidad Católica de Chile, junto a los doctores Héctor Croxatto y Joaquín Luco (ambos fueron "Premio Nacional de Ciencias"). Una vez ordenado sacerdote, dirigió su interés hacia los aspectos humanistas de la medicina, escribiendo en 1951 sus "Apuntes de Historia de la Medicina”, y, en 1953 su monografía "Ética Profesional".

\section{Referencias}

1- FYE WB. Ventricular fibrilation and defibrillation: historical perspectives with emphasis on the contributions of John MacWilliam, Carl Wiggers, and William Kouwenhoven. Circulation 1985;71:858-865. (Referencia recomendada, base de esta revisión).

2- WIGGERS CJ. The physiologic basis for cardiac resuscitation from ventricular fibrillation - method for serial defibrillation. Am Heart J 1940;20:413.
3- ZALAQUETT R. 50 años de cirugía de bypass coronario. Meditar el pasado, enfrentar el presente y forjar el futuro. Rev Chil Cardiol 2017;36:162-169.

4- WIGGERS CJ, WEGRIA R, PIÑERA B. The effects of myocardial ischemia on the fibrillation threshold - the mechanism of spontaneus ventricular fibrillation following coronary occlusion. Am Jour Physiol 1940;131:309. 